\title{
Transformation of Temporal Properties between Auditory Midbrain and Cortex in the Awake Mongolian Gerbil
}

\author{
Maria Ter-Mikaelian, ${ }^{1}$ Dan H. Sanes, ${ }^{1,2}$ and Malcolm N. Semple ${ }^{1,3}$ \\ ${ }^{1}$ Center for Neural Science and Departments of ${ }^{2}$ Biology and ${ }^{3}$ Psychology, New York University, New York, New York 10003
}

The neural representation of meaningful stimulus features is thought to rely on precise discharge characteristics of the auditory cortex. Precisely timed onset spikes putatively carry the majority of stimulus-related information in auditory cortical neurons but make a small contribution to stimulus representation in the auditory midbrain. Because these conclusions derive primarily from anesthetized preparations, we reexamined temporal coding properties of single neurons in the awake gerbil inferior colliculus (IC) and compared them with primary auditory cortex (AI). Surprisingly, AI neurons displayed a reduction of temporal precision compared with those in the IC. Furthermore, this hierarchical transition from high to low temporal fidelity was observed for both static and dynamic stimuli. Because most of the data that support temporal precision were obtained under anesthesia, we also reexamined response properties of IC and AI neurons under these conditions. Our results show that anesthesia has profound effects on the trial-to-trial variability and reliability of discharge and significantly improves the temporal precision of AI neurons to both tones and amplitude-modulated stimuli. In contrast, IC temporal properties are only mildly affected by anesthesia. These results underscore the pitfalls of using anesthetized preparations to study temporal coding. Our findings in awake animals reveal that AI neurons combine faster adaptation kinetics and a longer temporal window than evident in IC to represent ongoing acoustic stimuli.

Key words: inferior colliculus; auditory cortex; anesthetic effects; adaptation; amplitude modulation; binary coding

\section{Introduction}

Precise spike timing to the onset of an acoustic feature is thought to support many auditory percepts, including discrimination of consecutive sounds, the differentiation of phonemes in speech, and the spatial position of a sound source (Phillips and Farmer, 1990; Phillips and Hall, 1990; Eggermont, 1995; Furukawa and Middlebrooks, 2002; Stecker and Middlebrooks, 2003). Previously proposed temporal coding strategies require that spike latency remain consistent over multiple presentations of the same stimulus, in other words, that onset variability be relatively low (Heil, 2004). This criterion is satisfied by firing patterns in primary auditory cortex (AI), in which first-spike latency exhibits low variability that is comparable with that observed in auditory nerve fibers (Phillips and Hall, 1990). Indeed, there are reports that the precision of first-spike timing in AI can actually improve on the auditory nerve (Heil and Irvine, 1997). It has been proposed that such accuracy may be achieved in part by a secure and temporally precise synapse between the medial geniculate body and AI (Rose and Metherate, 2005). Additionally, precisely timed excitatory and inhibitory conductances that are cotuned for frequency and intensity can account for precise, transient responses

Received Nov. 7, 2006; revised April 27, 2007; accepted April 28, 2007.

This work was supported by National Institutes of Health Grant DC006864 (D.H.S.) and a grant from New York University (M.N.S.). We thank Dr. Shigeyuki Kuwada for helpful advice concerning the unanesthetized recording preparation and Kuei-Chu Chen for technical assistance.

Correspondence should be addressed to Malcolm N. Semple, Center for Neural Science, New York University, 4 Washington Place, Room 809, New York, NY 10003. E-mail: mal.semple@nyu.edu.

D01:10.1523/JNEUROSCI.4848-06.2007

Copyright $\odot 2007$ Society for Neuroscience $\quad$ 0270-6474/07/276091-12\$15.00/0 in AI (Wehr and Zador, 2003). However, such temporal coding schemes have been proposed primarily on the basis of anesthetized datasets.

It has been demonstrated that auditory cortical discharge patterns containing only the first spike may carry as much as $89 \%$ of the information about stimulus location available in the full spiking response (Furukawa and Middlebrooks, 2002). Additionally, mean first-spike latency was more informative than the average spike count or the mean spread of spikes within the response pattern. In the inferior colliculus (IC), conversely, first-spike timing made a relatively small contribution to encoding sound location, with substantial information carried by the rate and timing of discharges throughout the response (Chase and Young, 2006).

Our goal was to assess the evolution of temporal precision between two loci in the ascending auditory pathway in the awake gerbil. Surprisingly, we observed a decrease in temporal fidelity between the IC and AI in responses to either static or amplitudemodulated stimuli. A reexamination of temporal processing under anesthesia revealed significant anesthetic effects on temporal coding properties in AI but not IC. These effects have previously led to a different conclusion about hierarchical processing in the auditory system. We present a new interpretation of the IC-AI coding transformation. IC neurons exhibit greater temporal precision, slower adaptation kinetics, and a shorter "temporal window" (the time interval during which a neuron integrates input), allowing for the representation of continuous, rapidly modulated sounds. In contrast, the temporal response properties of AI neurons are better suited for representing slow temporal modulations in the acoustic environment. 


\section{Materials and Methods}

Animal preparation

All procedures relating to the maintenance and use of animals in this study were approved by the University Animal Welfare Committee at New York University.

Surgical preparation for chronic recordings. Adult gerbils were premedicated with ketoprophen $(1.5 \mathrm{mg} / \mathrm{kg}$, i.n. $)$ and dexamethasone $(0.35 \mathrm{mg} /$ $\mathrm{kg}$, i.p.) and hydrated with normosol (1.5 ml, s.c.). The animals were anesthetized with isoflurane, maintained by monitoring withdrawal reflexes and respiration rate. A small threaded metal post was positioned horizontally along the midline and secured with small bone screws and dental acrylic. A silver ground wire was secured in a small hole drilled into the anterior skull. For recording access to the IC, a plate of intraparietal bone was removed over the cerebellum (Spitzer and Semple, 1993). For AI recording, the craniotomy was performed over the left temporal cortex caudal to the bregma suture (Thomas et al., 1993). In both cases, the dura was left intact. A thin wall of dental cement was built along the perimeter of the craniotomy, and the enclosure was covered with silicone oil to maintain moisture and prevent surface edema. The craniotomized area was covered with a disposable cap of silicone elastomer (Sammons Preston Rolyan, Bolingbrook, IL).

Surgical preparation for acute recordings. Adult gerbils were anesthetized with a surgical dose of sodium pentobarbital ( $50 \mathrm{mg} / \mathrm{kg}$, i.p.) and ketamine $(30 \mathrm{mg} / \mathrm{kg}$, i.p.). A tracheotomy was performed to prevent airway obstruction during the experiment, and atropine $(0.08 \mathrm{mg} / \mathrm{kg}$, i.m.) was administered to minimize mucous secretions. Preparation of the craniotomy and head cap were similar to the procedures described above for unanesthetized recording. Throughout the experiment, the withdrawal reflex was monitored, and $20 \%$ supplemental doses of both sodium pentobarbital and ketamine were administered intramuscularly as needed. The body temperature of the animal was maintained at $37^{\circ} \mathrm{C}$ for the duration of the surgery and recording. The experiment was terminated by administrating an overdose of sodium pentobarbital $(>150$ $\mathrm{mg} / \mathrm{kg}$, i.p.).

For all recordings, the animal was placed in a soundproof chamber (Industrial Acoustics Company, Bronx, NY), and the head was stabilized using the head post. During chronic recording sessions, animals stood comfortably on a platform and were free to move their limbs while the head position remained fixed. If an animal exhibited heightened anxiety or restlessness, medetomidine (a relaxant that acts on $\alpha_{2}$-adrenoceptors) was administered intranasally at the beginning of the recording session at a dosage of one to three drops $(\sim 0.1-0.3 \mathrm{mg} / \mathrm{kg})$ and supplemented as necessary. It has been demonstrated that medetomidine lowers motivation but does not affect attention or response accuracy in rats performing a short-term memory task (Ruotsalainen et al., 1997). Similarly, gerbils trained to detect amplitude modulation in our laboratory are able to perform the task after medetomidine administration (D. H. Sanes, unpublished observations). An additional analysis not detailed in this report showed that key neural response properties [firing rate, minimum first-spike latency, and synchrony cutoff frequency for sinusoidally amplitude-modulated (SAM) tones remained stable with time elapsed after medetomidine administration (47-200 $\mathrm{min}$ ) in both IC and AI. Indeed, $91 \%$ of all cells (IC and AI) recorded in sessions in which medetomidine was used were obtained $60 \mathrm{~min}$ or later after the administration of the relaxant. Because medetomidine did not produce an observable effect on neuronal activity, the results for cells recorded with and without medetomidine use are presented together.

Nine animals (four IC and five AI) were used for acute recordings under anesthesia and 16 animals (four IC and $12 \mathrm{AI}$ ) were used for chronic awake recording experiments. One animal was excluded from the study because of a difference in the position of the craniotomy.

\section{Electrophysiological recording}

Tungsten electrodes (1.5-2.5 MW; MicroProbe, Gaithersburg, MD) were advanced ventrally through the craniotomy. For AI recording, an electrode tip angle of $\sim 14^{\circ}$ lateral to vertical was used; for IC recording, the electrode tip was angled $25-45^{\circ}$ anterior to vertical. Single-unit recording procedures were identical to those described previously (Malone and Semple, 2001). The number of recorded cells and their correspond-
A
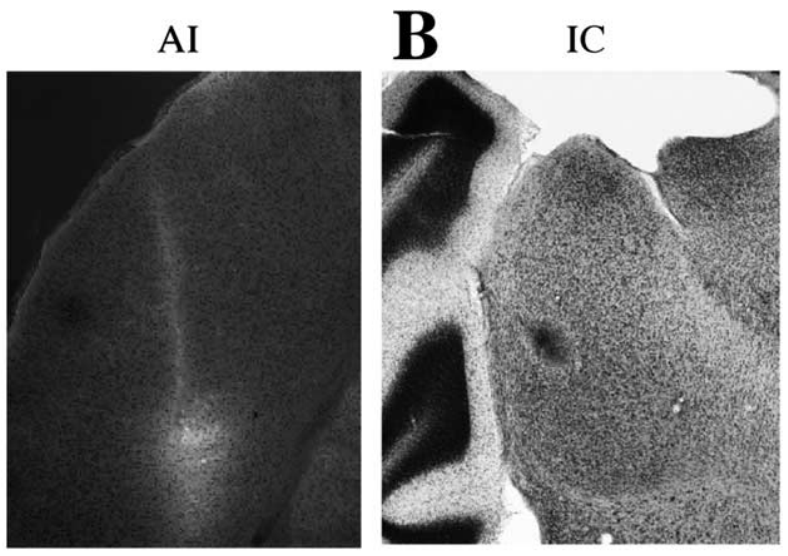

C

40 $\square$ awake AI

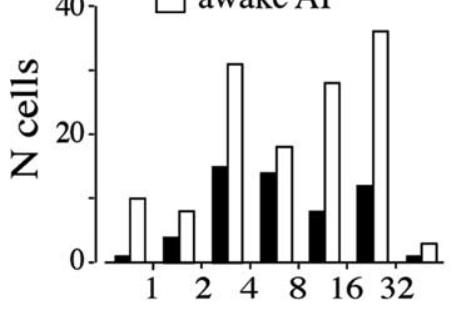

D

anesth IC awake IC

best frequency $(\mathrm{kHz})$

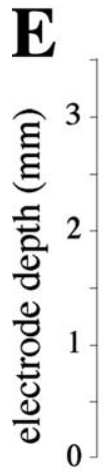

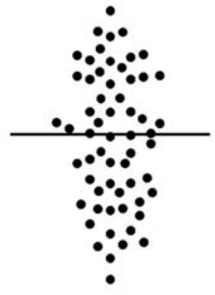

anesth AI

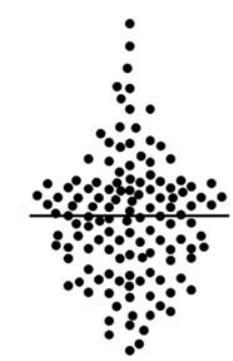

awake AI
Figure 1. Location and tuning characteristics of recording sites. $A$, Dark-field image of a coronal section through the left Al showing an electrode track in which a fluorescein injection was made. Dorsal is toward the top and lateral is to the left. B, Sagittal section through IC showing an electrode track terminating in a lesion site. Both sections are counterstained with cresyl violet. $\boldsymbol{C}, \boldsymbol{D}$, Histograms of BF of all recorded units in Al and IC. $\boldsymbol{E}$, Electrode insertion depths for recorded units in anesthetized and awake Al. Horizontal lines indicate population medians.

ing best frequencies are shown in Figure 1. AI recording depths were compared for anesthetized and awake preparations: electrode insertion distances spanned a range of $\sim 0.5-3 \mathrm{~mm}$, and population medians were not significantly different (Mann-Whitney $U$ test, two-tailed, $p=$ $0.1608 ; n=189$; median, $1.77 \mathrm{~mm}$ anesthetized and $1.63 \mathrm{~mm}$ awake) (Fig. 1E).

\section{Acoustic stimulation}

The system used for stimulus generation and sound delivery as well as the calibration procedure have been described previously (Malone and Semple, 2001). Acoustic stimuli were presented to each ear through an electrostatic speaker coupled to a custom-made ear insert. All stimuli were presented monaurally except in cases in which a monaural stimulus failed to elicit reliable responses, in which case a binaural stimulus was used. Sound pressure level (SPL) is expressed relative to $20 \mu \mathrm{Pa}$.

Each neuron was assessed with a battery of stimulus protocols de- 
Table 1. Numbers of cells whose response PSTHs to BF tones, noise, and $20 \mathrm{~Hz}$ SAM were fit with a decaying exponential function

\begin{tabular}{|c|c|c|c|c|c|c|c|c|c|}
\hline & \multicolumn{3}{|c|}{$2 \mathrm{sBF}$ tone } & \multicolumn{3}{|c|}{ 2s noise } & \multicolumn{3}{|c|}{10 s $20 \mathrm{~Hz}$ SAM } \\
\hline & Fit & Failed & Excluded & Fit & Failed & Excluded & Fit & Failed & Excluded \\
\hline Anesthetized IC & 16 & 2 & & & & & & & \\
\hline Anesthetized Al & 25 & 3 & & & & & & & \\
\hline Awake IC & 24 & 3 & 2 & 14 & 5 & 1 & 37 & 4 & 2 \\
\hline Awake Al & 40 & & & 33 & 1 & 1 & 37 & & \\
\hline
\end{tabular}

The first column under each stimulus type shows the number of cells for which successful fits were generated, the second column shows the number of cells for which fits did not converge, and the third column gives the number of cells excluded from the analysis as a result of having a buildup response pattern.

signed to determine its response properties for static and modulated sounds. Typically, the approximate frequency range was obtained with an iso-intensity function at $10-30 \mathrm{~dB}$ above threshold. This was followed by a rate-level function at the estimated best frequency (BF). The standard stimulus battery included a $2 \mathrm{~s}$ tone delivered at the BF of the unit, and a $2 \mathrm{~s}$ narrow-band white-noise stimulus bandpassed geometrically $(100 \%)$ about the BF. These long stimuli were shaped with a $5 \mathrm{~ms}$ cosineramped rise/fall time and were presented for at least 10 trials, with a $1 \mathrm{~s}$ intertrial interval. Brief tone and noise stimuli were identical to the longer stimuli, except that the duration was $50 \mathrm{~ms}$ and the intertrial interval was $950 \mathrm{~ms}$. Stimulus thresholds were determined separately for each stimulus type, and responses were characterized at $10 \mathrm{~dB}$ above threshold for noise stimuli and $15 \mathrm{~dB}$ above threshold for tones (or at the peak of a nonmonotonic rate-level function if this was $<15 \mathrm{~dB}$ above threshold).

SAM tones (10 s duration with $10 \mathrm{~ms}$ rise/fall) were presented at BF and at the SPL that produced the strongest synchronized response (often the same as the best SPL for tone stimulation). Modulation transfer functions (MTFs) were obtained by presenting two trials of multiple modulation frequencies at $100 \%$ modulation depth with an intertrial interval of $2 \mathrm{~s}$. The following modulation frequencies were typically presented to each AC neuron: $0.1,0.5,1,2,3,5,10,20,30,50$, and $100 \mathrm{~Hz}$. For IC neurons, 200, 300, and $400 \mathrm{~Hz}$ were also presented. If a neuron exhibited statistically significant synchrony to the modulation period at the highest modulation rate $(100 \mathrm{~Hz}$ for $\mathrm{AC}$ and $400 \mathrm{~Hz}$ for IC), the modulation frequency was tested in additional $100 \mathrm{~Hz}$ increments until a cutoff frequency was obtained. An unmodulated tone at the same frequency and SPL and of the same duration as the SAM tones was included in the stimulus sequence as a control. For some cells, MTFs were also obtained for $400 \mathrm{~ms}$ SAM tones with a $10 \mathrm{~ms}$ rise time and an intertrial interval of $600 \mathrm{~ms}$. Twenty trials were presented at each modulation frequency. In addition, the spontaneous firing rate and pattern of each cell was recorded during a continuous $30 \mathrm{~s}$ silent period.

\section{Data analysis}

Spike times were stored and viewed as raster plots or peristimulus time histograms (PSTHs). Minimum first-spike latency was determined at BF and at the SPL that produced the earliest response by selecting the first peak in the PSTH to a $200 \mathrm{~ms}$ tone (binned by $10 \mathrm{~ms}$ ) as the region of interest (ROI). The corresponding raster plot was used to determine the mean onset time of the first spike over 10 or more trials. Using the same ROI, the SD of the first-spike time over all trials ("SD of the minimum latency") was calculated to provide an estimate of the temporal precision of the response onset. As an additional measure of temporal fidelity of the response onset, a $20 \mathrm{~ms}$ time window was positioned over the raster plot such that it contained the first spike of as many trials as possible. Trials that contained no spike in this time period were counted as "misses," and the proportion of misses over the total number of trials was determined.

To analyze the kinetics of the firing response decay in response to long constant stimuli, the peristimulus time histogram of the response of each unit to a $2 \mathrm{~s}$ BF tone or noise burst or a $10 \mathrm{~s}$ SAM tone was fit with an exponential decay function of the form $Y(t)=A e^{-k t}+P$, where $Y(t)$ is the firing rate at time $t, k$ is the inverse of the decay time constant, $A$ is a magnitude scaling factor, and $P$ is the plateau firing rate. The resulting fit equation allowed us to interpolate the time at which the response declined to half and a quarter of the initial peak and also to estimate firing at set time points during the stimulus presentation. Additionally, popu- lation medians of the fit parameters $A, k$, and $P$ were used to create typical response profiles for IC and AI neurons with and without anesthesia. Table 1 summarizes the number of cells used in the adaptation analysis from each population. In several cases, curve fit generation failed because the regression algorithm did not converge on best-fit values. Generally, this occurred when the number of spikes was insufficient to constrain the fit to a single solution. Additionally, several cells were excluded from the adaptation analysis because they had a buildup response pattern.

Average firing rates to tones and noise bursts were calculated over a time window equal to the stimulus duration but offset from the stimulus start time by the latency of the unit. Curve fits of spiking responses to tones and noise bursts were based on peristimulus time histograms, generated by sliding a $50 \mathrm{~ms}$ summation window in steps of $25 \mathrm{~ms}$ (equivalent to smoothing a histogram with $50 \mathrm{~ms}$ bins). For fitting responses to $10 \mathrm{~s}$ SAM tones, a $100 \mathrm{~ms}$ summation window was moved in steps of 50 ms. The peak response was defined as the largest bin in the histogram, which usually occurred near the onset of the stimulus. If the peak response occurred within the first seven bins of the histogram, the bins preceding it were excluded before curve fitting. This ensured that the fit best reflected the decaying phase of the response.

Responses to SAM tones were analyzed for firing rate and synchrony to the stimulus period. The ROI excluded the first $100 \mathrm{~ms}$ to ensure that the response to the modulation was examined without contamination by the onset response (Liang et al., 2002). Average firing rate over the entire ROI was determined for each modulation frequency and used to construct a rate MTF (rMTF). Additionally, a period histogram was created on a time base equal to the duration of one period for each modulation rate (100 bins per period). This period histogram was used to calculate a vector strength value at each modulation frequency, such that a temporal MTF (tMTF) could be constructed. Additionally, a modified Rayleigh statistic, $z=2 \times N \times \mathrm{VS}^{2}$, where $N$ is the number of spikes in the ROI and VS is the vector strength, was used to determine the level of significance of the vector strength measurement (Liang et al., 2002). Vector strength values corresponding to a modified Rayleigh measure of 13.8 were considered statistically significant at a $0.1 \%$ confidence level. Therefore, a SAM synchrony cutoff frequency was determined by interpolating within the Rayleigh measure curve to the modulation frequency associated with this value.

The temporal precision and coherence of the periodic response to SAM tones was assessed by summing period histograms of the responses of individual neurons to a $2 \mathrm{~Hz}$ SAM stimulus (binned by $10 \mathrm{~ms}$, excluding the first $100 \mathrm{~ms}$ of each trial) to create a pooled period histogram for each of the four study populations. Each pooled period histogram was normalized to account for differences in sample size and maximum firing rate. All neurons displaying significant synchronization to SAM at $2 \mathrm{~Hz}$ were included in the analysis (34 anesthetized IC, 26 anesthetized AI, 41 awake IC, and 41 awake AI).

For both the tMTF and the rMTF, a best modulation frequency (BMF) was determined as the tested frequency that yielded the highest average firing rate (for $\mathrm{rBMF}$ ) or the tested frequency that yielded the highest vector strength with statistical significance at the $p<0.001$ level (for tBMF).

\section{Histology}

In all experiments, the location of the electrode in $\mathrm{AI}$ or in the IC central nucleus was determined based on a tonotopic progression matching those described previously for gerbil AI (Thomas et al., 1993) and IC (Malone and Semple, 2001). In some but not all animals, a representative recording site was marked by an electrolytic lesion or injection of fluorescein or biocytin $(5 \%$ in $0.9 \% \mathrm{NaCl})$. For dye injection, the tungsten recording electrode was withdrawn and a glass electrode was inserted in the same location. After death, animals were perfused intracardially with $0.9 \% \mathrm{NaCl}$, followed by $0.4 \mathrm{~m}$ paraformaldehyde. The brain was refrigerated in $0.4 \mathrm{M}$ paraformaldehyde for several days and then saturated in a 
$30 \%$ sucrose solution and sectioned coronally (AI) or sagittally (IC) on a freezing microtome. Sections stained with biocytin were reacted by a conventional $\mathrm{ABC}-\mathrm{DAB}$ protocol. In addition, all sections were counterstained with cresyl violet. An example of a fluorescein injection site in AI and an electrolytic lesion in IC is shown in Figure 1.

\section{Results}

Results are presented from 41 single units in anesthetized IC, 55 in anesthetized AI, 76 in awake IC, and 120 in awake AI.

\section{Temporal precision in auditory cortex is lower than in IC}

We examined the temporal precision of discharges to an unmodulated stimulus in IC and AI of awake gerbils. To do this, we compared the first-spike timing variability (the SD of the latency; see Materials and Methods) in response to $200 \mathrm{~ms}$ BF tones. In Figure 2, example responses are shown in $A$ and $B$, and $C$ plots the population results. Surprisingly, the AI neuron population shows significantly higher first-spike timing variability compared with IC (Mann-Whitney $U$ test, two-tailed, $p=0.0479 ; n=113$ ), indicating a loss of temporal precision in the ascending auditory pathway. The median values (indicated in Fig. $2 C$ by horizontal black bars) were $2.5 \mathrm{~ms}$ for AI and $1.85 \mathrm{~ms}$ for IC.

Improved temporal precision in $\mathrm{AI}$ is an artifact of anesthesia This finding was paradoxical given previous reports of improved temporal precision in AI compared with subcortical auditory nuclei (Phillips and Hall, 1990; Heil and Irvine, 1997). Because previous observations were based on recordings in anesthetized preparations, we decided to duplicate our experiment in anesthetized gerbils to test the possibility that the increased temporal precision was attributable to anesthetic effects. Figure $3 \mathrm{~A}$ shows the tone response of an individual anesthetized AI cell; the onset of spiking is highly precise across periods, and the first-spike latency is short compared with a typical awake AI cell (Fig. 2A). Figure $3 B$ plots the population data with mean minimum latency on the abscissa and SD on the ordinate. Remarkably, anesthesia reduces the median minimum latency in AI by 12 ms (MannWhitney $U$ test, one-tailed, $p<0.0001 ; n=96$ ). Furthermore, under anesthesia, the response onset becomes more precisely timed (Fig. 3C) (Mann-Whitney $U$ test, one-tailed, $p=0.0005$; $n=96$ ). Although some overlap is observed in the distributions, there is a short-latency, low-variability population in anesthetized AI that is unmatched in the awake group. Thus, improved temporal precision in AI appears to be an artifact of anesthesia. Conversely, in IC, anesthetic use appears to have no effect on either the minimum latency (Mann-Whitney $U$ test, two-tailed, $p=0.2265 ; n=95$ ) or the SD of the minimum latency (MannWhitney $U$ test, two-tailed, $p=0.4207 ; n=95$ ) (Fig. 4).

It has frequently been observed that cortical neurons under anesthesia tend to fire one or no spikes on each stimulus presentation (DeWeese et al., 2003). We wondered whether the increased precision of response onset in anesthetized AI was accompanied by an increase in the number of missed trials. For each AI cell, we calculated the proportion of trials in which no spikes were fired during a $20 \mathrm{~ms}$ window containing the shortestlatency spikes (see Materials and Methods) (an example of the analysis is shown in Fig. $5 A, B$ ). The majority of awake AI units $(61.8 \%, 21$ of 34$)$ exhibited reliable response onsets on every trial, whereas $>75 \%$ of anesthetized AI units (31 of 41) missed firing an onset spike during one or more trials (Fig. $5 \mathrm{C}$ ). Although stimulus level was set relative to the threshold of each cell, the result cannot be attributed to differences in tone level because thresholds were very similar in the two populations (Mann-

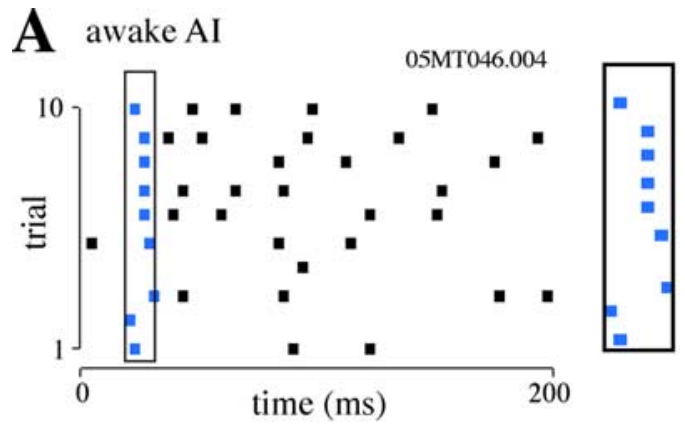

B awake IC
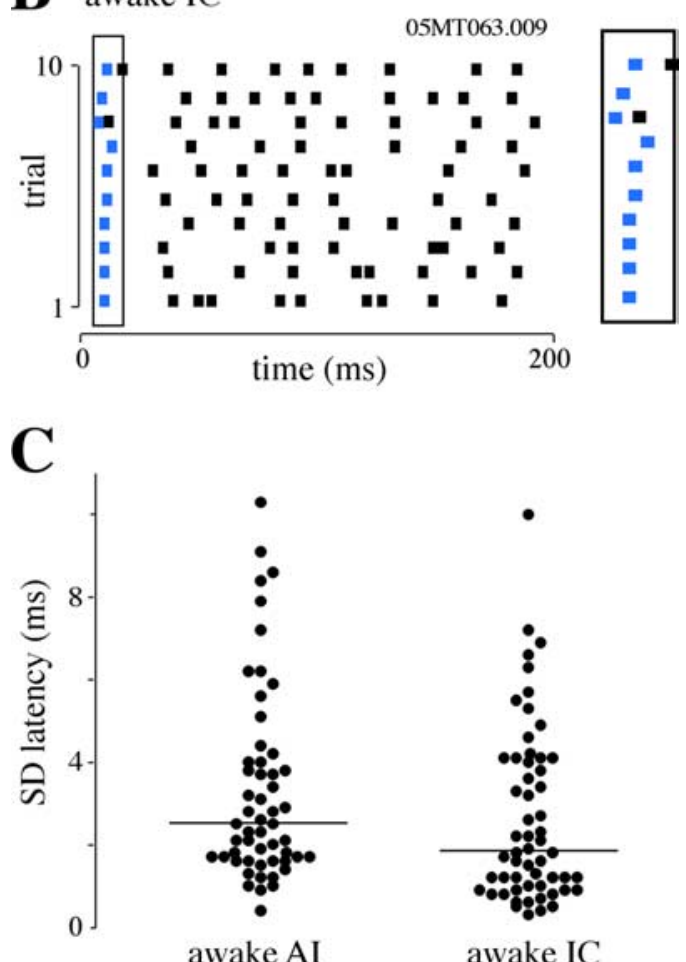

awake IC

Figure 2. First-spike timing is less precise in Al than in IC. $A, B$, Rasters of responses of an example awake $\mathrm{Al}(\boldsymbol{A})$ and awake IC $(\boldsymbol{B})$ cell to a $200 \mathrm{~ms} B$ b tone. Windowed portions indicate the $\mathrm{ROl}$ for latency analysis, shown expanded on the right. The first spike in the ROl in each trial is shown in blue. C, Population plot of SD of the minimum latency in response to BF tone pips in $\mathrm{Al}$ and IC.

Whitney $U$ test, two-tailed, $p=0.996 ; n=78$ ). Because anesthetized AI cells generally fired only an onset spike or burst on each presentation of the stimulus, trials with no spikes within the 20 ms analysis window generally meant trials in which no spikes were fired at all, i.e., those in which the cell failed to signal the presence of the stimulus. This suggests that the apparent high temporal precision of anesthetized AI responses comes at the cost of low fidelity, complicating the interpretation of the output of each cell.

\section{Temporal response profiles differ dramatically between IC} and $\mathrm{AI}$

We have shown that the temporal characteristics of the early phase of the response of AI cells can be strongly affected by anesthesia and that these effects may obscure the response transformation between IC and AI. Are additional differences in the kinetics of IC and AI cell responses to long-lasting continuous stimuli confounded by anesthetic state? The response kinetics 
A anesth AI

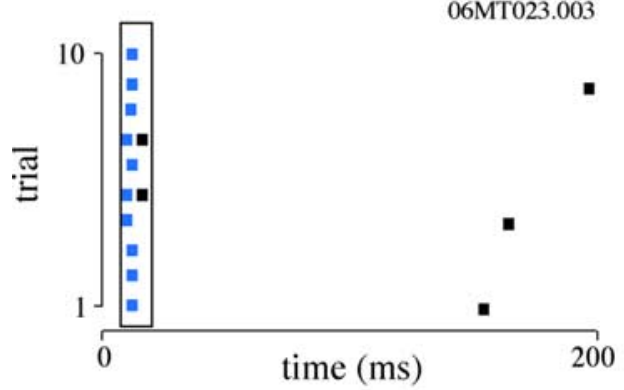

B
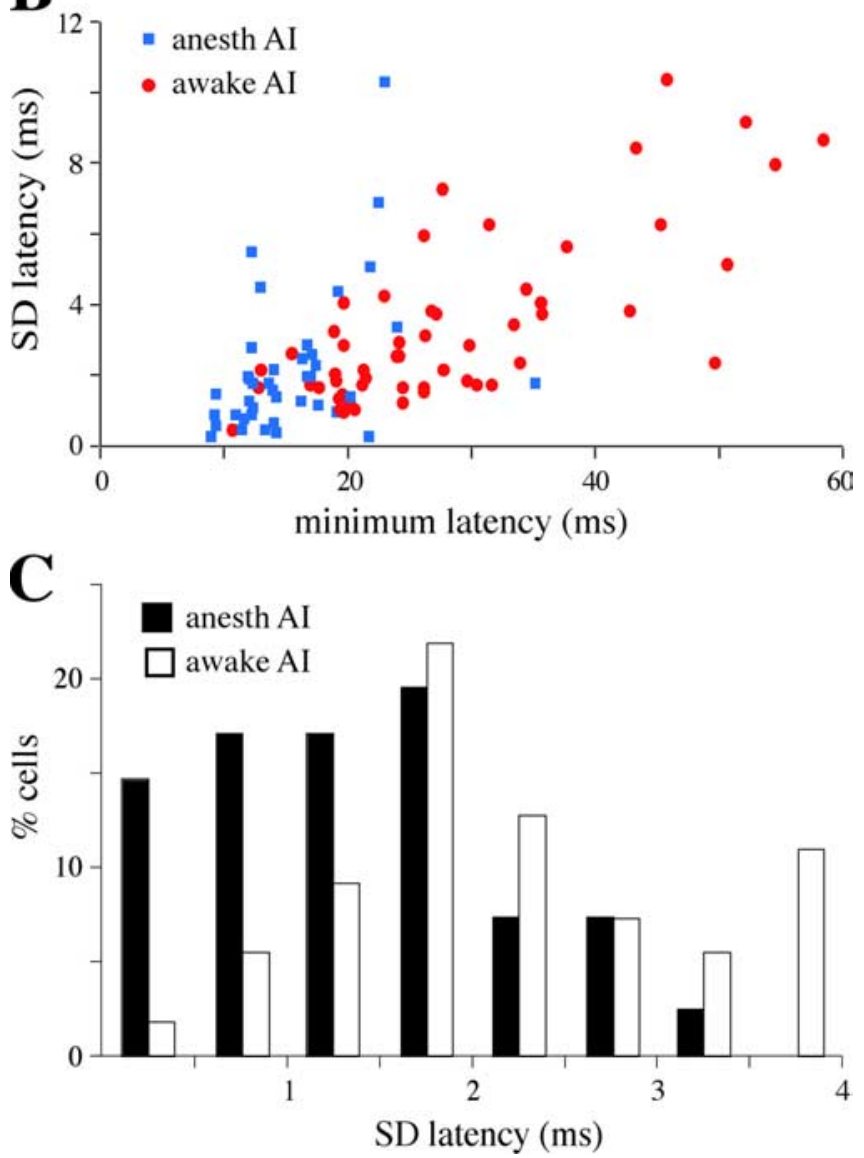

Figure 3. Al first-spike latency and latency variability are reduced by anesthesia. $\boldsymbol{A}, \mathrm{Re}-$ sponse of an example anesthetized Al cell to a $200 \mathrm{~ms}$ BF tone; conventions as in Figure 2. $\boldsymbol{B}$, Population plot of minimum first-spike latency and $S D$ of the minimum latency in response to $B F$ tone pips in anesthetized and awake Al. C, Histogram of SD of the latency in anesthetized and awake Al. Filled bars indicate anesthetized Al, and open bars indicate awake Al. Only values up to $4 \mathrm{~ms}$ are shown to highlight the difference between the populations.

over a longer time period were examined by fitting a decaying exponential function to the PSTH for a $2 \mathrm{~s}$ tone (the PSTH of a typical awake AI cell is shown in Fig. 6A). This generated fit parameters $A, k$, and $P$ for each cell, corresponding to a magnitude scaling factor, the inverse of the decay time constant, and the plateau firing rate, respectively (see Materials and Methods). We used the median values for each population to plot a "prototype" response adaptation profile for a typical anesthetized AI and a typical awake AI cell (Fig. 6C, gray lines). Clearly, the response profiles diverge considerably in both magnitude and shape: the anesthetized and awake AI cell populations differ significantly in all three fit parameters (Mann-Whitney $U$ test, two-tailed; $A, p=$
A anesth IC
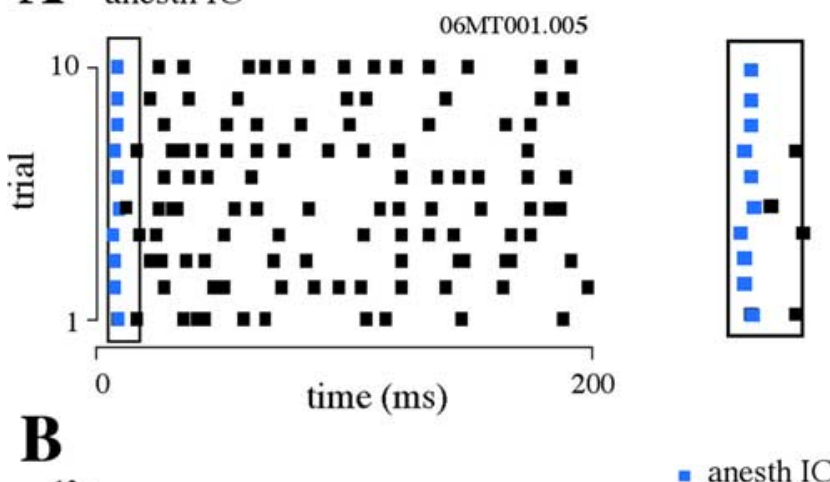

- anesth IC
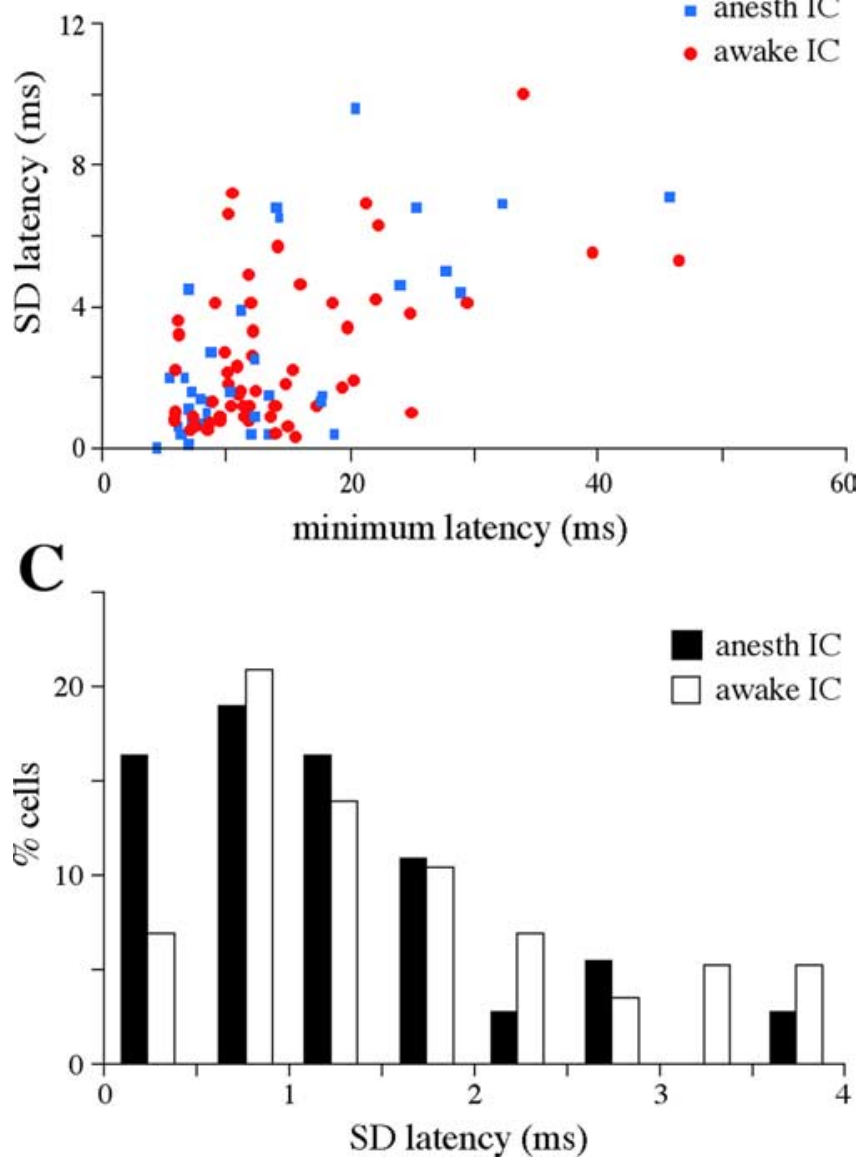

Figure 4. IC latency variability is unaffected by anesthesia. $A$, Response of an example anesthetized IC cell to a $200 \mathrm{~ms}$ BF tone. $B$, Population plot of minimum first-spike latency and SD of the minimum latency in response to BF tone pips in anesthetized and awake IC. C, Histogram of SD of the latency in anesthetized and awake IC. Conventions as in Figure 3.

$0.0006, k, p=0.0385, P, p=0.0091 ; n=65)$. The median awake AI cell fires at a rate of 37.2 spikes/s within the first $50 \mathrm{~ms}$, and its response declines to a plateau of 0.4 spikes/s by the end of the $2 \mathrm{~s}$ stimulus; the median anesthetized AI cell, in contrast, progresses from an initial firing rate of 22 spikes/s to a plateau of 1.3 spikes/s. The time constant of firing rate decay is also significantly faster in awake AI cells, with a median value corresponding to $73 \mathrm{~ms}$ compared with $181 \mathrm{~ms}$ for anesthetized AI cells.

Thus, AI response kinetics in the awake state are indeed substantially different from those predicted by anesthetized data. We analyzed the IC temporal response profile using the same method (the response of an example cell is shown in Fig. $6 \mathrm{~B}$ ). Interestingly, both the initial response level and the rate of decay are very 


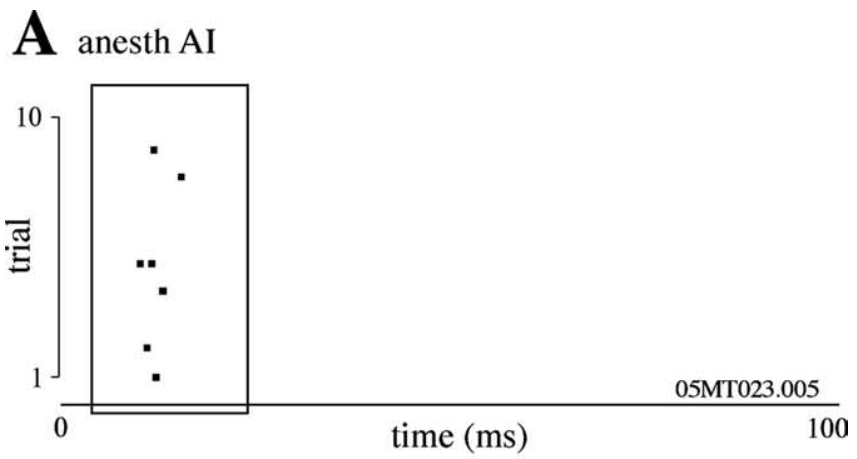

B awake AI

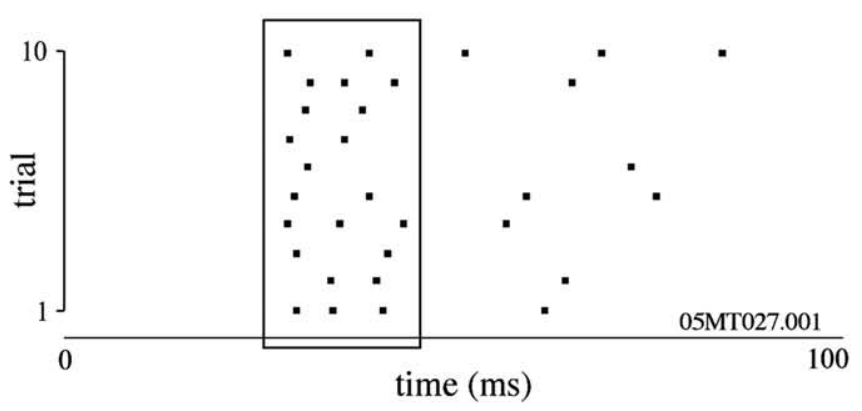

C

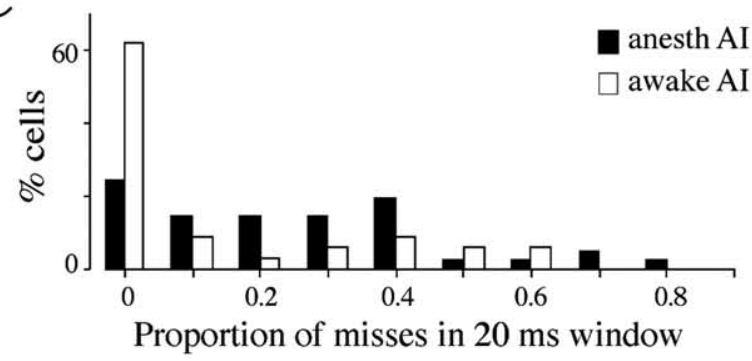

D

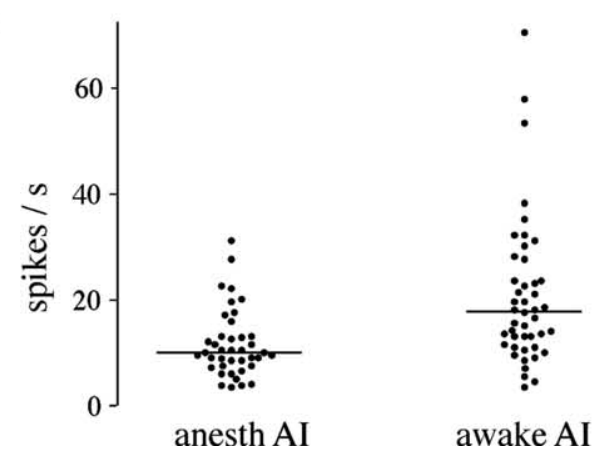

Figure 5. Al tone responses are more reliable in awake animals. $A, B$, Rasters of the first 100 ms of the responses of an example anesthetized $(\boldsymbol{A})$ and awake $(\boldsymbol{B})$ Al cell to a $200 \mathrm{~ms} B$ b tone. Windowed portions indicate the $20 \mathrm{~ms}$ ROI for determining the number of missed trials. $\boldsymbol{C}$, Histogram of the proportion of missed trials in anesthetized and awake Al cells. Filled bars indicate anesthetized $\mathrm{Al}$, and open bars indicate awake AI. D, Population plot of average firing rate for a $200 \mathrm{~ms}$ BF tone in anesthetized and awake Al. Horizontal lines indicate population medians.

similar with and without anesthesia; however, in anesthetized IC, the firing rate adaptation continues for a longer period (Fig. 6C, black lines). In this case, of the three fit parameters, only the plateau response magnitude differed significantly between anesthetized and awake IC (Mann-Whitney $U$ test, one-tailed, $p=$
A awake AI

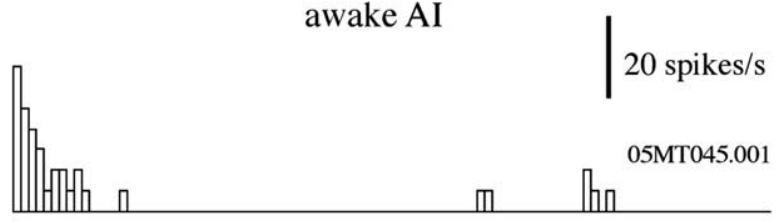

B

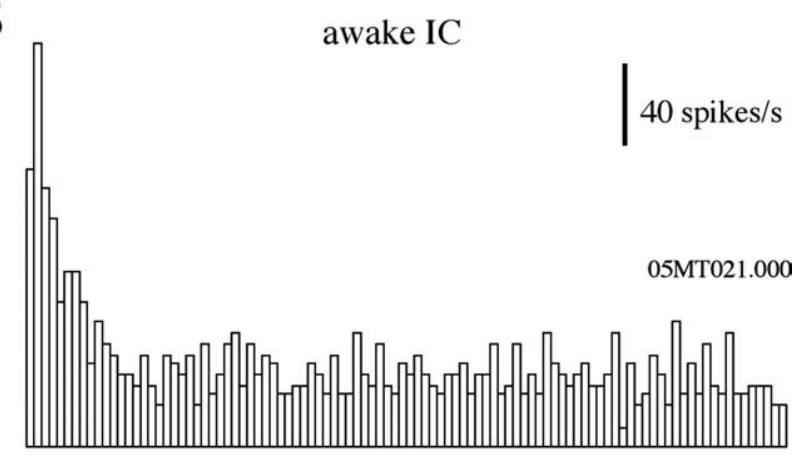

C

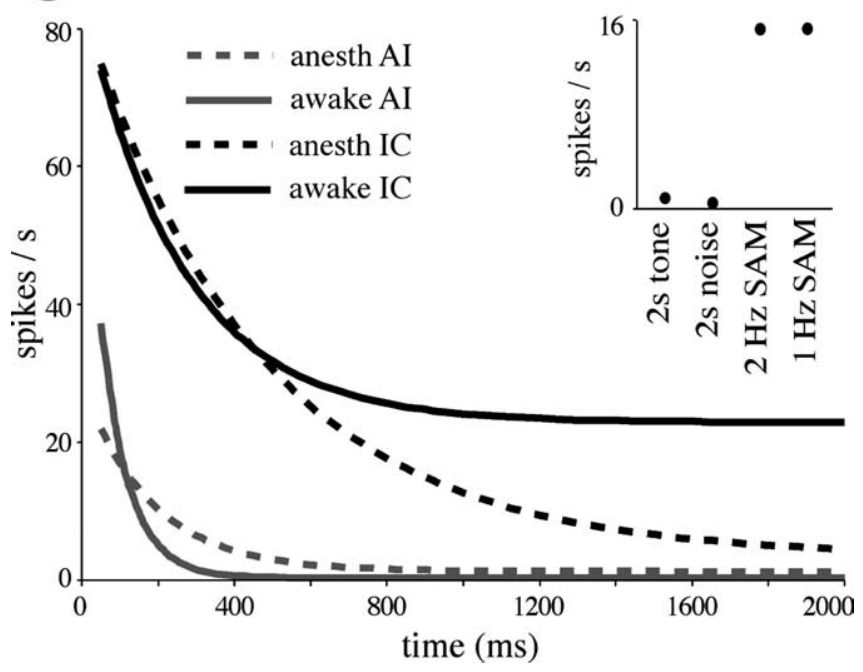

Figure 6. Temporal response profile to long constant stimuli in Al and IC. $A, B$, PSTH of the response of an example awake $\mathrm{Al}(\boldsymbol{A})$ and awake IC $(\boldsymbol{B})$ cell to 10 trials of a $2 \mathrm{~S} B$ B tone. $\boldsymbol{C}$, Response adaptation profiles in response to a $2 \mathrm{~s}$ BF tone for a typical cell in Al (gray lines) and IC (black lines), obtained from the population median parameters of an exponential decay curve fit to the PSTH of each cell. The curve begins at 50 ms to match the corresponding PSTH. Solid lines denote awake populations, and dashed lines denote anesthetized populations. Inset, Plateau firing rates for an example awake Al cell for four different types of continuous stimuli, from exponential decay curve fits.

$0.0016 ; n=40$ ), corresponding to a steady-state firing rate of 3.5 and 22.9 spikes/s, respectively.

A direct comparison of response adaptation kinetics in IC and $\mathrm{AI}$ in the awake gerbil reveals a substantial coding transformation (Fig. 6C). In addition to responding with an initial firing rate equal to approximately half of that in IC, AI neurons exhibit a response that declines significantly faster ( $k$, Mann-Whitney $U$ test, two-tailed, $p=0.0369 ; n=64)$ to a much lower steady-state firing level $(P$, Mann-Whitney $U$ test, two-tailed, $p<0.0001 ; n=$ $64)$. The median values of $k$ correspond to decay time constants of $257 \mathrm{~ms}$ for IC and $73 \mathrm{~ms}$ for AI neurons. The median plateau firing rates, as mentioned above, are 22.9 and 0.4 spikes/s for IC and AI cells, respectively. Comparing the curves in Figure $6 \mathrm{C}$ 
reveals that anesthesia reduces the apparent difference in temporal response profiles between IC and AI by lowering the steadystate firing rate of IC cells and increasing that of AI cells and by slowing adaptation in AI cells, thus obscuring the hierarchical transformation.

Interestingly, when a $2 \mathrm{~s}$ broadband noise burst stimulus is used instead of a $\mathrm{BF}$ tone, similar response adaptation is observed (data not shown). By $1.9 \mathrm{~s}$ after stimulus onset, the spike rate in the median awake IC cell declines to $\sim 25 \%$ of the initial peak response, whereas the median awake AI neuron fires at $\sim 1 \%$ of its initial spike rate (Mann-Whitney $U$ test, one-tailed, $p<$ $0.0001 ; n=47)$, similar to 31 and $1 \%$ in a typical IC and AI cell, respectively, for a $2 \mathrm{~s} \mathrm{BF}$ tone (Fig. $6 \mathrm{C}$ ). Likewise, the firing response of an IC cell to a long (10 s) $20 \mathrm{~Hz}$ SAM tone settles to a plateau at $\sim 30 \%$ of the initial spike rate compared with $\sim 4 \%$ for an AI cell, as determined by a decaying exponential fit (data not shown; Mann-Whitney $U$ test, one-tailed, $p<0.0001 ; n=74$ ). As with an unmodulated tone, the firing rate in response to a $10 \mathrm{~s}$ SAM tone declines faster in AI cells than in IC cells (data not shown; time to $50 \%$ of the initial firing rate: Mann-Whitney $U$ test, one tailed, $p<0.0001 ; n=51$ ). Thus, response adaptation kinetics in each structure appear to be very similar across a wide range of long, continuous stimuli and differ significantly between $\mathrm{IC}$ and $\mathrm{AI}$.

It should be noted that, for individual AI cells, adaptation kinetics can vary significantly depending on how well the stimulus is optimized for the particular cell (Wang et al., 2005). Figure $6 C$, inset, shows a comparison of steady-state firing rates of an example awake AI neuron for four different stimulus types, two of which are long, static stimuli as discussed above, and two of which are SAM tones with modulation frequencies in the range most effective for AI. Whereas plateau firing rates for the unmodulated tone and noise stimuli are near 0 , a robust response is maintained for low-frequency SAM even after $10 \mathrm{~s}$ of stimulation.

\section{Temporal responses to a dynamic stimulus transform greatly between IC and AI}

We tested whether a hierarchical transformation also occurs in the temporal response profile to a dynamic stimulus modulated on a shorter timescale. We presented a series of SAM tones at different modulation frequencies and examined the ability of individual neurons to respond reliably at the same phase of every period (a quality that we termed "synchronization" to the stimulus). Long (10 s) stimuli were chosen to separate onset effects from ongoing temporal properties. First, we investigated the effects of anesthetic state on synchronization. Under anesthesia, a much larger proportion of AI neurons failed to show statistically significant synchrony (see Materials and Methods) to SAM at any of the tested modulation frequencies (36\% in anesthetized AI vs $9 \%$ in unanesthetized AI). However, of the neurons responding synchronously to at least one modulation rate, a greater percentage continued to display significant synchrony to modulation rates above $5 \mathrm{~Hz}$ in the anesthetized AI population (Fig. 7D). This appears to be another example of improved temporal regularity in anesthetized AI. However, a greater proportion of awake AI cells than anesthetized AI cells synchronized to modulation rates below $2 \mathrm{~Hz}$ (Fig. 7D), and the corresponding vector strength values were generally much higher in the awake state (data not shown). For example, at $2 \mathrm{~Hz}$ the median vector strength for awake AI cells was 0.73 compared with 0.43 for anesthetized AI (Mann-Whitney $U$ test, one-tailed, $p<0.0001 ; n=70$ ).

In the IC, nearly all cells synchronized to at least one SAM frequency in both awake and anesthetized conditions (97.7 and
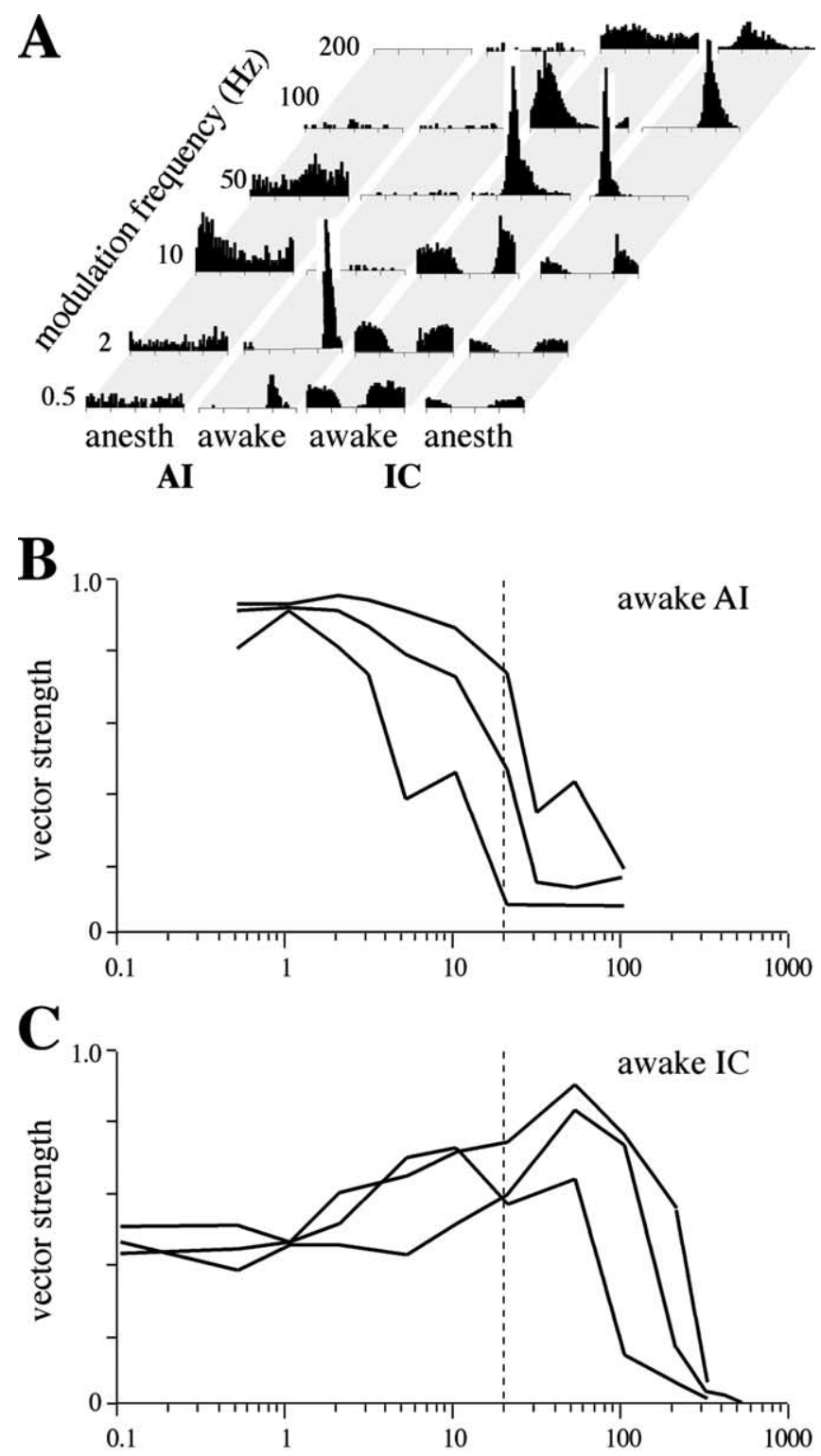

D

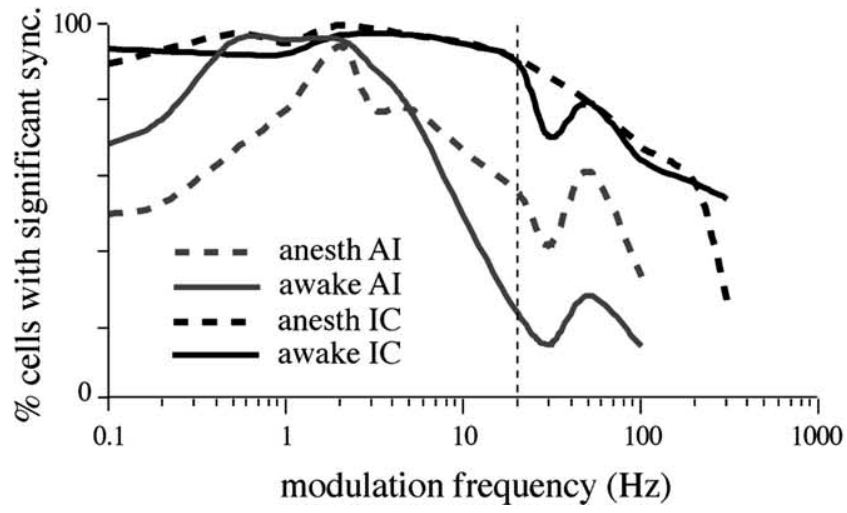

Figure 7. Temporal representation of envelope modulations in Al and IC. $\boldsymbol{A}$, Period histograms of responses of four example cells, one from each study group, to six SAM frequencies. Normalization was performed separately for AI and IC. $\boldsymbol{B}, \boldsymbol{C}$, tMTFs of three representative awake $A I(B)$ and IC (C) cells. A vertical line has been added to each graph at $20 \mathrm{~Hz}$ to facilitate comparison. D, Percentage of cells in Al (gray lines) and IC (black lines) showing significant synchrony (Rayleigh test, $p<0.001$ ) to the period of SAM as a function of the modulation frequency. Solid lines denote awake and dashed lines denote anesthetized populations. 
$94.5 \%$, respectively). No difference was observed in the ability of these cells to fire synchronously to modulations at different rates (Fig. 7D).

In the awake state, the temporal characteristics of SAM responses in IC and AI are strikingly different. Figure 7 shows examples of tMTFs of individual AI (Fig. 7B) and IC (Fig. 7C) neurons, generated by stimulating with 10 -s-long SAM tones at different modulation frequencies and calculating a vector strength value for each. A vertical line has been added along the $20 \mathrm{~Hz}$ value on the abscissa for ease of comparison. It can be observed in these examples that, although tMTFs vary within each structure, IC tMTFs tend to be more bandpass and "roll over" at higher frequencies than those of AI cells. This difference in synchronization limit is very pronounced at the population level (Fig. 7D). We quantified this phenomenon by determining the synchrony cutoff frequency of each neuron tested with SAM in awake animals (see Materials and Methods). As expected, the median cutoff frequency for IC neurons $(190 \mathrm{~Hz})$ was significantly higher than for AI neurons $(7 \mathrm{~Hz}$ ) (Mann-Whitney $U$ test, one-tailed, $p<0.0001 ; n=88)$. To determine whether the synchronization limits of neurons were correlated with their response latency, we plotted these variables against one another (Fig. 8A). A negative relationship was observed in both the IC and AI population.

For some awake IC cells, we also tested brief SAM tones (400 $\mathrm{ms}$ ) and compared the synchrony cutoff frequency for $400 \mathrm{~ms}$ and $10 \mathrm{~s}$ SAM stimuli. In most neurons, altering stimulus duration caused a change in the synchrony cutoff estimate; however, the direction of change varied across cells, and the overall range of cutoff values remained very similar (Fig. $8 B$ ).

For a more detailed study of the way in which IC and AI represent modulations on the timescale important for speech (Houtgast and Steeneken, 1985; Shannon et al., 1995), we chose a low-frequency $(2 \mathrm{~Hz}$ ) SAM stimulus. For each cell, we folded the firing response to a $10 \mathrm{~s}$ SAM tone on the $500 \mathrm{~ms}$ period to create a period histogram; period histograms of all neurons in each of the four study populations were then summed to create a normalized pooled period histogram for each group. Figure $9 \mathrm{~A}$ shows the results for anesthetized and awake AI. The distribution of spikes within the period is clearly peaked for both populations; however, the temporal pattern of discharge differs qualitatively. As a population, anesthetized AI neurons respond earlier in the period of the modulation, and the highest firing occurs early in the response, at $\sim 60^{\circ}$ phase. By comparison, maximum firing in awake AI occurs nearly one-quarter of a period later, at $\sim 140^{\circ}$. An earlier local peak in firing does occur in awake AI at $\sim 75^{\circ}$ phase; however, this peak is significantly smaller, suggesting a less coherent onset in the response to the modulation period and a slower rise to maximal response than in anesthetized AI. This parallels the observation that first spikes in response to tones occur earlier and are more precisely timed in anesthetized AI than in awake AI. Interestingly, in the anesthetized AI population, spikes are distributed more uniformly across the modulation period than in awake AI. This is primarily a result of poorer synchronization of individual anesthetized AI cells to the stimulus envelope, as evident from their relatively low vector strength values compared with awake AI (see above). In the latter, maximal firing occurs closer to the actual maximum of the stimulus envelope, and the difference between the lowest and highest firing in the period is much more pronounced, suggesting that awake AI more faithfully represents the shape of the modulation. This distinction, combined with the relatively high percentage of nonsynchronizing units under anesthesia, makes anesthetized AI output
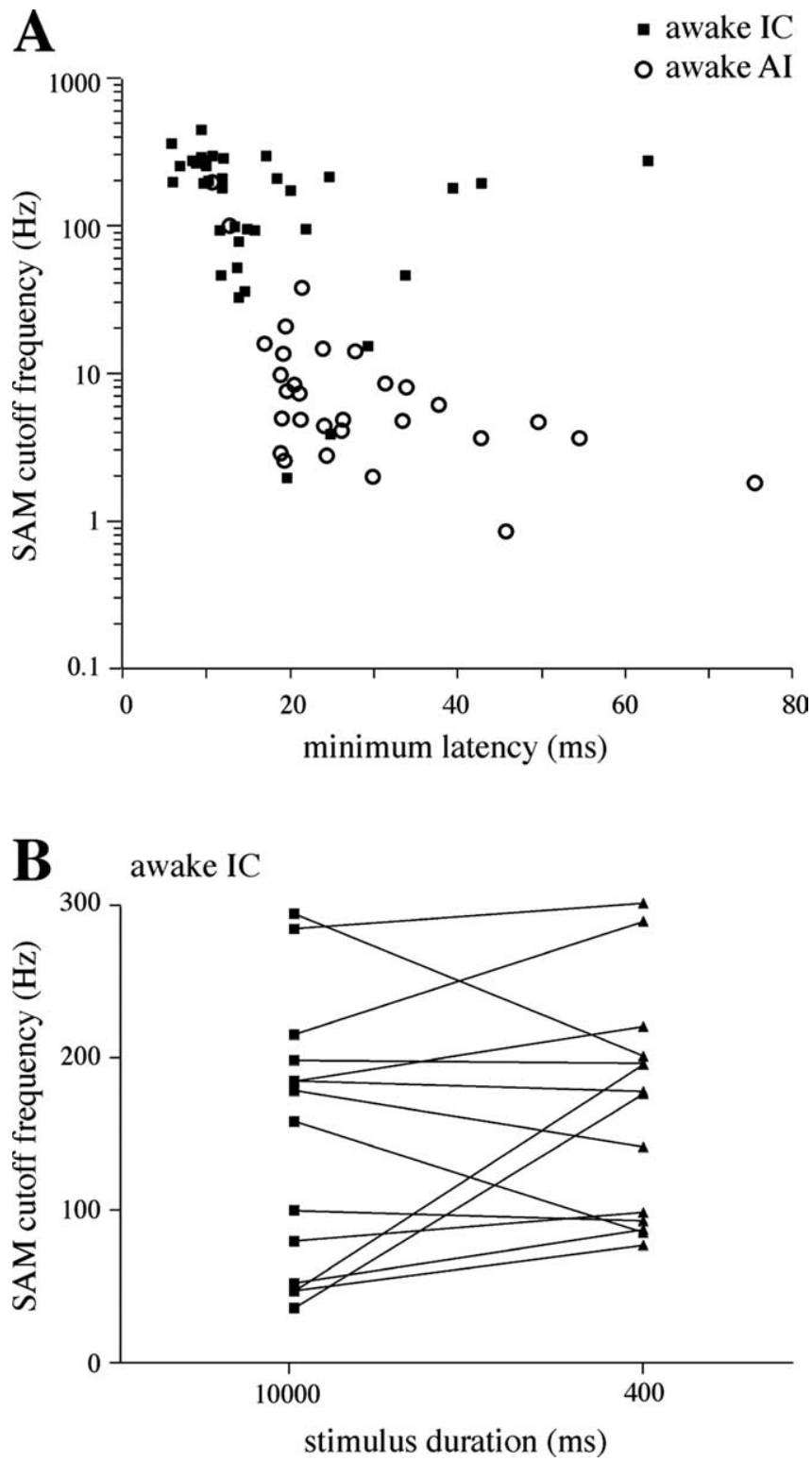

Figure 8. SAM tMTF cutoff is correlated with minimum latency and depends on stimulus duration. $\boldsymbol{A}$, Individual cells in awake IC (filled squares) and AI (open circles). The sample is a subset of the full data because it was not always possible to obtain a reliable measure of minimum latency and SAM cutoff frequency in the same cell. B, Synchrony cutoff in 14 awake IC cells as assessed with $10 \mathrm{~s}$ and $400 \mathrm{~ms}$ SAM tones.

less informative about the sound stimulus than the output of awake AI.

In IC, the difference between anesthetized and awake discharge patterns is less dramatic (Fig. 9B). The shapes of the pooled period histograms are very similar, although the maximal response in anesthetized IC occurs slightly earlier, and more adaptation is observed during the second half of the period.

\section{Is there a rate-coding transformation between IC and AI?}

The previous sections have dealt with a temporal coding transformation between IC and AI and have shown how its interpretation has been complicated by anesthetic involvement. Others have demonstrated that firing rates in AI can also be strongly affected by anesthesia (Gaese and Ostwald, 2001; Wang et al., 2005), and this could serve as a confounding factor for a ratecoding transformation between IC and AI. We therefore exam- 

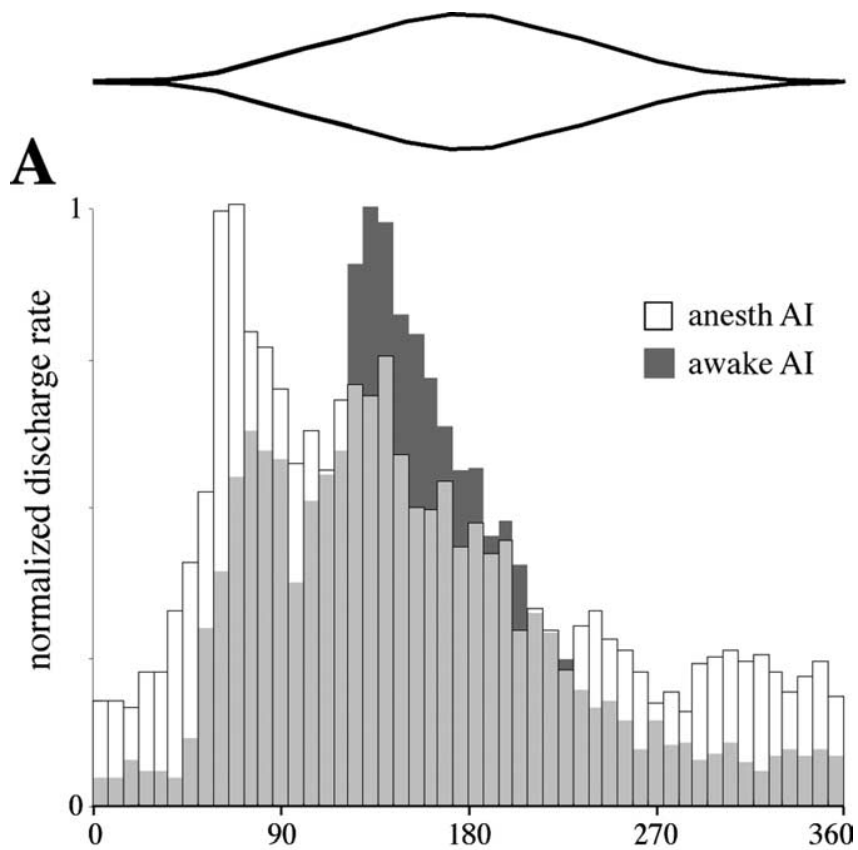

B

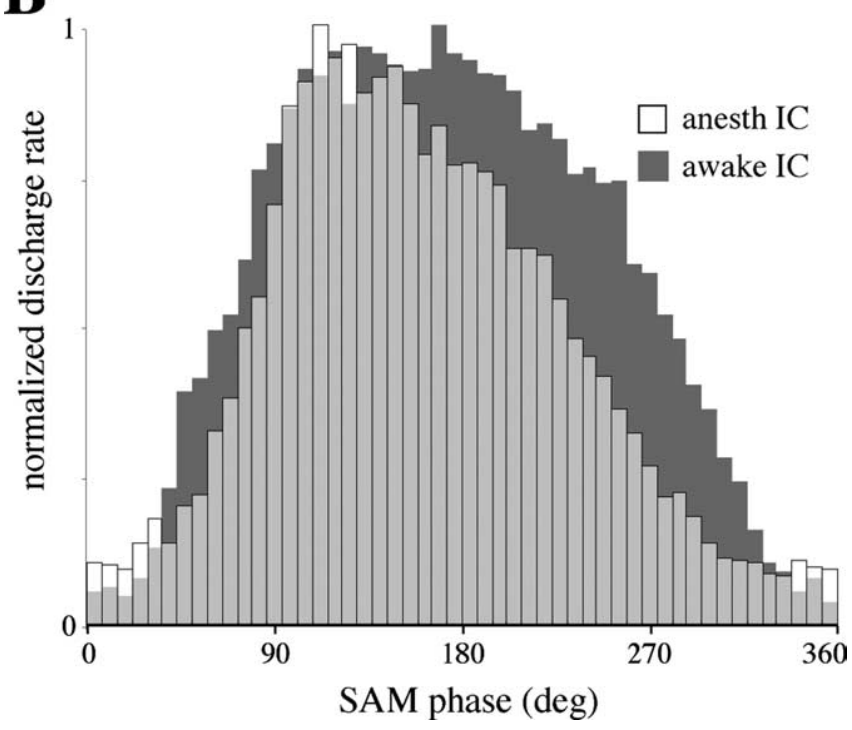

Figure 9. Discharge precision to the SAM envelope in Al and IC. Pooled period histograms of the responses of all tested $\mathrm{AI}(\boldsymbol{A})$ and IC $(\boldsymbol{B})$ neurons to the period of a 10-s-long $2 \mathrm{~Hz}$ SAM tone. The stimulus envelope is indicated at the top. Awake data are represented by shaded bars, and anesthetized data are represented by semitransparent white bars, which are superimposed. Histograms were generated by adding the period histograms of individual cells in each population and normalizing. Values along the abscissa correspond to modulation phase, in which one $500 \mathrm{~ms}$ period equals $360^{\circ}$.

ined stimulus-evoked firing rates in both structures with and without anesthetics.

Average firing rates to a $50 \mathrm{~ms}$ BF tone pip in awake and anesthetized AI constitute overlapping populations; however, responses in awake AI cells are significantly greater (Mann-Whitney $U$ test, two-tailed, $p=0.0006 ; n=72$; median values, 18 spikes/s for anesthetized and 34 spikes/s for awake AI; data not shown). A similar trend is observed with 200-ms-long stimuli (Mann-Whitney $U$ test, one-tailed, $p=0.0001 ; n=86$ ) (Fig. $5 D$ ). Conversely, no significant difference in firing rate was found between anesthetized and awake IC for either $50 \mathrm{~ms}$ (MannWhitney $U$ test, one-tailed, $p=0.3948 ; n=39$; data not shown) or $200 \mathrm{~ms}$ tones (Mann-Whitney $U$ test, one-tailed, $p=0.1405$; $n=89$; data not shown). Interestingly, any differences in soundevoked discharge rate between our awake and anesthetized preparations cannot be attributed to an overall reduction in excitability: no significant difference in spontaneous firing rate was observed with anesthetic state in either IC (Mann-Whitney $U$ test, one-tailed, $p=0.2161 ; n=94$; median, 3.8 spikes/s anesthetized and 2.4 awake; data not shown) or AI (Mann-Whitney $U$ test, one-tailed, $p=0.1343 ; n=74$; median, 1.6 spikes/s anesthetized and 1.0 awake; data not shown).

In the absence of the suppressive effects of anesthetic, a substantial firing rate difference persists between IC and AI cells. In part, this distinction is amplified by the different adaptation kinetics in these structures; although in all cases, the IC neuron population tends to fire more strongly, the difference is more pronounced for longer stimuli (data not shown). Population comparisons of average firing rate for all three tested tone durations ( $50 \mathrm{~ms}, 200 \mathrm{~ms}$, and $2 \mathrm{~s}$ ) show IC and AI to be significantly different (50 ms: Mann-Whitney $U$ test, one-tailed, $p=0.0289$, $n=59 ; 200 \mathrm{~ms}:$ Mann-Whitney $U$ test, one-tailed, $p<0.0001$, $n=95 ; 2$ s: Mann-Whitney $U$ test, one-tailed, $p<0.0001, n=$ 71). A similar trend was observed with broadband noise burst stimulation (data not shown): although responses to $50 \mathrm{~ms}$ stimuli are significantly stronger in IC neurons than in AI cells (Mann-Whitney $U$ test, one-tailed, $p=0.0085 ; n=59$ ), an even greater difference in firing rate was observed with $2 \mathrm{~s}$ noise bursts (Mann-Whitney $U$ test, one-tailed, $p<0.0001 ; n=95$ ).

We also examined the modulation of firing rate as a function of SAM frequency. A best modulation frequency according to spike rate (rBMF) was determined for each cell and compared with its best modulation frequency for synchrony, or tBMF (see Materials and Methods). In awake IC neurons, no significant difference was observed between rBMF and tBMF values (data not shown; paired $t$ test, one-tailed, $p=0.2326 ; n=41$ ), and the population means were 50 and $35 \mathrm{~Hz}$, respectively. For awake AI cells, the rBMF tended to be somewhat higher than the tBMF (data not shown; paired $t$ test, one-tailed, $p=0.0265 ; n=49$ ), but both numbers (means, 6.4 and $1.9 \mathrm{~Hz}$, respectively) were low relative to those of IC neurons, as expected. Interestingly, in the anesthetized AI, rBMFs were slightly higher than in awake AI, mirroring the shift in synchrony limits (Mann-Whitney $U$ test, two-tailed, $p=0.0425 ; n=78$; medians, $3.0 \mathrm{~Hz}$ anesthetized and $2.0 \mathrm{~Hz}$ awake AI). For the marmoset, it has been suggested that AI cells that fail to respond synchronously to modulating stimuli may nonetheless represent them with a rate code (Lu et al., 2001; $\mathrm{Lu}$ and Wang, 2004). In our sample in gerbil, nonsynchronizing AI neurons either responded to SAM with a brief onset burst and remained essentially quiescent throughout the duration of the stimulus or failed to respond at all.

\section{Discussion}

In awake animals, AI cells display higher variability than IC cells in signaling the beginning of a new stimulus or a new cycle in an ongoing modulation. Anesthetics significantly alter the temporal response profiles of AI cells to static as well as modulated stimuli but produce minor effects in IC. Data obtained from anesthetized preparations suggest an IC-AI coding transformation toward temporally precise, binary-like responses signaling the onsets of new stimuli or stimulus cycles; in contrast, recordings in the awake state suggest a quantitative rather than qualitative difference in response timing variability, adaptation kinetics, and envelope-tracking limits between IC and AI. 


\section{Temporal coding in awake IC and AI}

Although temporally precise onset spikes have frequently been viewed as a critical feature of cortical coding (Furukawa and Middlebrooks, 2002; DeWeese et al., 2003; Heil, 2004), the significance of temporal fidelity in AI has been recently reexamined. An information-theoretic analysis of AI responses in awake cats showed that response patterns containing only the first spike conveyed $<50 \%$ of the stimulus-related information available in the full spiking response (Mickey and Middlebrooks, 2003). Moreover, shuffled spike patterns missing all temporal components also lost $\sim 50 \%$ of stimulus-related information (Mickey and Middlebrooks, 2003). We demonstrate that, although awake AI responses exhibit less precisely timed onsets than those of anesthetized AI, they occur more reliably and create a more faithful representation of the ongoing stimulus envelope at low frequencies.

We show that AI responses to long, continuous stimuli exhibit substantially lower initial amplitudes, more rapid decay kinetics, and a lower steady-state firing level than those of IC cells. Although little systematic information is available on response adaptation kinetics in unanesthetized IC and AI, our results are consistent with two previous studies. The first (Goldstein et al., 1968 ) reported that $\sim 80 \%$ of $\mathrm{AI}$ units in awake cat responded transiently to $100 \mathrm{~ms}$ tones. The second study (Chimoto et al., 2002 ) found that $38 \%$ of awake cat AI cells exhibited sustained responses to $500 \mathrm{~ms}$ tones, $22 \%$ adapted significantly within 500 ms, and 15\% responded only with onset and/or offset spikes. Unlike the present study, neither report considered response decay beyond $500 \mathrm{~ms}$.

A striking observation is the similarity in the temporal profiles of responses to long-duration tones, noise, and SAM within each structure. This suggests a slow, stimulus-invariant temporal filter, with faster kinetics and more extensive filtering in AI (median time constants, $257 \mathrm{~ms}$ in IC and $73 \mathrm{~ms}$ in AI). This filter may gate the appearance of spikes to stimulus features occurring on a faster timescale, such as amplitude modulations in $20 \mathrm{~Hz}$ SAM, which IC neurons track with synchronous spikes.

In addition to a slow filter controlling response adaptation, our findings indicate a filtering process operating on a more rapid timescale. Responses to SAM suggest that IC can temporally track stimulus changes occurring at a rate of up to $190 \mathrm{~Hz}$, whereas AI can do so up to only $7 \mathrm{~Hz}$. These limits are broadly consistent with those reported previously for anesthetized and awake IC (Batra et al., 1989; Krishna and Semple, 2000) and for awake AI (Creutzfeldt et al., 1980; Malone et al., 2000; Lu et al., 2001; Liang et al., 2002). AI synchrony cutoffs in this study are somewhat lower than those reported by others; this may reflect species differences or perhaps the shorter stimuli in previous studies (Lu et al., 2001; Liang et al., 2002) meant that synchrony was assessed before responses reached a steady state. However, although we observed an effect of stimulus duration on SAM synchrony cutoffs in awake IC, the direction of change was not systematic across our sample. Our findings suggest that neurons in IC and AI have a temporal window of 5 and $143 \mathrm{~ms}$, respectively, corresponding to the inverse of the synchrony cutoff frequency.

\section{Anesthetic effects on temporal coding}

The barbiturate-ketamine anesthetic regimen in this study was chosen as representative of previous investigations in auditory cortical and collicular physiology. Pentobarbital prolongs the open time of GABA-activated chloride channels (Nicoll et al., 1975; MacDonald et al., 1989), whereas ketamine is a noncompetitive NMDA receptor antagonist (Franks and Lieb, 1994) that also reduces AMPA-mediated depolarization (Leong et al., 2004). Partial substitution of ketamine for barbiturate is advantageous, because ketamine is believed to be less suppressive of neural activity (Astl et al., 1996; Wehr and Zador, 2005).

A difference in minimum response latency between anesthetized and unanesthetized cortical neurons has not been noticed previously, but our results are consistent with two previous reports on gerbil auditory cortex. Single and multiunit response latencies in anesthetized gerbil AI reportedly range between 10 and $25 \mathrm{~ms}$ for AI and the anterior auditory field (AAF) (Thomas et al., 1993). By comparison, the same group of investigators later reported that multiunit latencies in fields AI and AAF of unanesthetized gerbils varied from 5 to $65 \mathrm{~ms}$, with $\sim 50 \%$ exceeding 25 ms (Schulze et al., 1997). Because multiunit recordings tend to favor the shortest latency neuron, it is likely that response latencies in AI of awake animals are indeed significantly longer.

One potential explanation for this phenomenon is that different neural populations are sampled in recordings from anesthetized and unanesthetized animals. However, our distributions of BFs and thresholds indicate that the two populations are closely matched.

Another possibility is that an anesthesia-sensitive inhibitory mechanism ordinarily blocks depolarization and prevents early spikes in unanesthetized cortex. The disappearance of early inhibition under anesthesia may be attributable to pentobarbitalmediated $\mathrm{GABA}_{\mathrm{A}}$ receptor desensitization (Brownell et al., 1979; Wu and Oertel, 1986; Wu and Kelly, 1994; Feng et al., 2004). Indeed, certain thalamically activated neurons in layer $\mathrm{V}$ of rat auditory cortical slices reportedly exhibit fast inhibition (Hefti and Smith, 2000, 2003). Additionally, fast-spiking (presumably inhibitory) interneurons in thalamorecipient layers III/IV of mouse auditory cortex exhibit faster EPSPs than regular-spiking (presumed excitatory) neurons in the same layers (Rose and Metherate, 2005). These interneurons are well positioned to block onset responses of cells to which they project. Whereas layer IV neurons mainly project to layer III, layer III sends axons to all other cortical layers, including layer V (Mitani et al., 1985). Although recordings from awake animals limit the ability to localize individual recorded neurons, it is plausible that a significant proportion of recordings in both anesthetized and unanesthetized preparations were obtained in cortical layers other than layer IV.

A final possibility is that the latency difference between anesthetized and unanesthetized animals arises subcortically but above the level of the inferior colliculus, and thus its mechanism could not be observed in this preparation.

The relatively higher limits of response synchronization to SAM in the anesthetized cortex are consistent with the shift toward shorter latencies. A similar relationship between response latency and synchronization is evident for trains of repeating tones in rat auditory cortex (Kilgard and Merzenich, 1999) and for envelope modulation in marmoset cortex (Liang et al., 2002). However, anesthetized AI responses synchronized to amplitude modulations exhibited low vector strength, reflecting spikes scattered throughout the modulation period and unmatched in successive trials. Similarly, unreliable, irregular discharges characterized all but the onsets of responses to long tones in anesthetized $\mathrm{AI}$, resulting in an altered adaptation profile under anesthesia. We also observed that a large number of cells became unresponsive to SAM under anesthesia, which is consistent with strong, long-lasting inhibition such as may be induced by pentobarbital. The large number of missed trials in response to tones in anesthetized AI is also indicative of overall suppression. Finally, average discharge rates are significantly reduced by anesthesia in AI 
(Gaese and Ostwald, 2001; Wang et al., 2005) but not in IC. Because our analysis showed that the IC response profile decays further under anesthesia, some reduction in average firing rate relative to awake IC is expected; however, it is not significant at the population level. The anesthetic regimen used in our study may account for the relatively minor anesthetic effects on IC response properties compared with some previous studies $(\mathrm{Ku}-$ wada et al., 1989; Tollin et al., 2004).

In conclusion, cortical neurons are ideally suited to represent biologically relevant sounds. They temporally encode lowfrequency modulations that predominate in natural sounds, including speech (Singh and Theunissen, 2003). Interestingly, they sacrifice some precision in representing acoustic fine structure to reliably encode the sound envelope. This is well matched by the fact that speech perception is quite robust to disturbances in fine structure but depends critically on the amplitude envelope (Drullman, 1995; Shannon et al., 1995). Although it is known that temporal fine structure is important in some speech reception situations such as in noisy environments and for tonal languages (Zeng et al., 2005; Kong and Zeng, 2006), our study only addresses envelope processing. Cortical neurons in the awake state represent not only the periodicity but the shape of the modulation envelope, which is an important cue for successful speech perception (Goswami et al., 2002).

\section{References}

Astl J, Popelar J, Kvasnak E, Syka J (1996) Comparison of response properties of neurons in the inferior colliculus of guinea pigs under different anesthetics. Audiology 35:335-345.

Batra R, Kuwada S, Stanford TR (1989) Temporal coding of envelopes and their interaural delays in the inferior colliculus of the unanesthetized rabbit. J Neurophysiol 61:257-268.

Brownell WE, Manis PB, Ritz LA (1979) Ipsilateral inhibitory responses in the cat lateral superior olive. Brain Res 177:189-193.

Chase SM, Young ED (2006) Spike-timing codes enhance the representation of multiple simultaneous sound-localization cues in the inferior colliculus. J Neurosci 26:3889-3898.

Chimoto S, Kitama T, Qin L, Sakayori S, Sato Y (2002) Tonal response patterns of primary auditory cortex neurons in alert cats. Brain Res 934:34-42.

Creutzfeldt O, Hellweg FC, Schreiner C (1980) Thalamocortical transformation of responses to complex auditory stimuli. Exp Brain Res 39:87-104.

DeWeese MR, Wehr M, Zador AM (2003) Binary spiking in auditory cortex. J Neurosci 23:7940-7949.

Drullman R (1995) Temporal envelope and fine structure cues for speech intelligibility. J Acoust Soc Am 97:585-592.

Eggermont JJ (1995) Representation of a voice onset time continuum in primary auditory cortex of the cat. J Acoust Soc Am 98:911-920.

Feng HJ, Bianchi MT, Macdonald RL (2004) Pentobarbital differentially modulates alpha1beta3delta and alpha1beta3gamma2L GABAA receptor currents. Mol Pharmacol 66:988-1003.

Franks NP, Lieb WR (1994) Molecular and cellular mechanisms of general anaesthesia. Nature 367:607-614.

Furukawa S, Middlebrooks JC (2002) Cortical representation of auditory space: information-bearing features of spike patterns. J Neurophysiol $87: 1749-1762$.

Gaese BH, Ostwald J (2001) Anesthesia changes frequency tuning of neurons in the rat primary auditory cortex. J Neurophysiol 86:1062-1066.

Goldstein Jr MH, Hall II JL, Butterfield BO (1968) Single-unit activity in the primary auditory cortex of unanesthetized cats. J Acoust Soc Am 43:444-455.

Goswami U, Thomson J, Richardson U, Stainthorp R, Hughes D, Rosen S, Scott SK (2002) Amplitude envelope onsets and developmental dyslexia: a new hypothesis. Proc Natl Acad Sci USA 99:10911-10916.

Hefti BJ, Smith PH (2000) Anatomy, physiology, and synaptic responses of rat layer $\mathrm{V}$ auditory cortical cells and effects of intracellular GABA(A) blockade. J Neurophysiol 83:2626-2638.

Hefti BJ, Smith PH (2003) Distribution and kinetic properties of GABAergic inputs to layer $\mathrm{V}$ pyramidal cells in rat auditory cortex. J Assoc Res Otolaryngol 4:106-121.

Heil P (2004) First-spike latency of auditory neurons revisited. Curr Opin Neurobiol 14:461-467.

Heil P, Irvine DR (1997) First-spike timing of auditory-nerve fibers and comparison with auditory cortex. J Neurophysiol 78:2438-2454.

Houtgast T, Steeneken HJM (1985) A review of the MTF concept in room acoustics and its use for estimating speech intelligibility in auditoria. J Acoust Soc Am 77:1069-1077.

Kilgard MP, Merzenich MM (1999) Distributed representation of spectral and temporal information in rat primary auditory cortex. Hear Res 134:16-28.

Kong YY, Zeng FG (2006) Temporal and spectral cues in Mandarin tone recognition. J Acoust Soc Am 120:2830-2840.

Krishna BS, Semple MN (2000) Auditory temporal processing: responses to sinusoidally amplitude-modulated tones in the inferior colliculus. J Neurophysiol 84:255-273.

Kuwada S, Batra R, Stanford TR (1989) Monaural and binaural response properties of neurons in the inferior colliculus of the rabbit: effects of sodium pentobarbital. J Neurophysiol 61:269-282.

Leong D, Puil E, Schwarz D (2004) Ketamine blocks non- $N$-methyl-Daspartate receptor channels attenuating glutamatergic transmission in the auditory cortex. Acta Otolaryngol 124:454-458.

Liang L, Lu T, Wang X (2002) Neural representations of sinusoidal amplitude and frequency modulations in the primary auditory cortex of awake primates. J Neurophysiol 87:2237-2261.

Lu T, Wang X (2004) Information content of auditory cortical responses to time-varying acoustic stimuli. J Neurophysiol 91:301-313.

Lu T, Liang L, Wang X (2001) Temporal and rate representations of timevarying signals in the auditory cortex of awake primates. Nat Neurosci 4:1131-1138.

MacDonald RL, Rogers CJ, Twyman RE (1989) Barbiturate regulation of kinetic properties of the GABAA receptor channel of mouse spinal neurones in culture. J Physiol (Lond) 417:483-500.

Malone BJ, Semple MN (2001) Effects of auditory stimulus context on the representation of frequency in the gerbil inferior colliculus. J Neurophysiol 86:1113-1130.

Malone BJ, Scott BH, Semple MN (2000) Processing of sinusoidal modulations of amplitude and frequency in the auditory cortex of alert rhesus macaques. Soc Neurosci Abstr 26:358.18.

Mickey BJ, Middlebrooks JC (2003) Representation of auditory space by cortical neurons in awake cats. J Neurosci 23:8649-8663.

Mitani A, Shimokouchi M, Itoh K, Nomura S, Kudo M, Mizuno N (1985) Morphology and laminar organization of electrophysiologically identified neurons in the primary auditory cortex in the cat. J Comp Neurol 235:430-447.

Nicoll RA, Eccles JC, Oshima T, Rubia F (1975) Prolongation of hippocampal inhibitory postsynaptic potentials by barbiturates. Nature 258:625-627.

Phillips DP, Farmer ME (1990) Acquired word deafness, and the temporal grain of sound representation in the primary auditory cortex. Behav Brain Res 40:85-94.

Phillips DP, Hall SE (1990) Response timing constraints on the cortical representation of sound time structure. J Acoust Soc Am 88:1403-1411.

Rose HJ, Metherate R (2005) Auditory thalamocortical transmission is reliable and temporally precise. J Neurophysiol 94:2019-2030.

Ruotsalainen S, Haapalinna A, Riekkinen Sr PJ, Sirvio J (1997) Dexmedetomidine reduces response tendency, but not accuracy of rats in attention and short-term memory tasks. Pharmacol Biochem Behav 56:31-40.

Schulze H, Ohl FW, Heil P, Scheich H (1997) Field-specific responses in the auditory cortex of the unanaesthetized Mongolian gerbil to tones and slow frequency modulations. J Comp Physiol A Neuroethol Sens Neural Behav Physiol 181:573-589.

Shannon RV, Zeng FG, Kamath V, Wygonski J, Ekelid M (1995) Speech recognition with primarily temporal cues. Science 270:303-304.

Singh NC, Theunissen FE (2003) Modulation spectra of natural sounds and ethological theories of auditory processing. J Acoust Soc Am 114:3394-3411.

Spitzer MW, Semple MN (1993) Responses of inferior colliculus neurons to 
time-varying interaural phase disparity: effects of shifting the locus of virtual motion. J Neurophysiol 69:1245-1263.

Stecker GC, Middlebrooks JC (2003) Distributed coding of sound locations in the auditory cortex. Biol Cybern 89:341-349.

Thomas H, Tillein J, Heil P, Scheich H (1993) Functional organization of auditory cortex in the mongolian gerbil (Meriones unguiculatus). I. Electrophysiological mapping of frequency representation and distinction of fields. Eur J Neurosci 5:882-897.

Tollin DJ, Populin LC, Yin TC (2004) Neural correlates of the precedence effect in the inferior colliculus of behaving cats. J Neurophysiol 92:3286-3297.

Wang X, Lu T, Snider RK, Liang L (2005) Sustained firing in auditory cortex evoked by preferred stimuli. Nature 435:341-346.
Wehr M, Zador AM (2003) Balanced inhibition underlies tuning and sharpens spike timing in auditory cortex. Nature 426:442-446.

Wehr M, Zador AM (2005) Synaptic mechanisms of forward suppression in rat auditory cortex. Neuron 47:437-445.

Wu SH, Kelly JB (1994) Physiological evidence for ipsilateral inhibition in the lateral superior olive: synaptic responses in mouse brain slice. Hear Res 73:57-64.

Wu SH, Oertel D (1986) Inhibitory circuitry in the ventral cochlear nucleus is probably mediated by glycine. J Neurosci 6:2691-2706.

Zeng FG, Nie K, Stickney GS, Kong YY, Vongphoe M, Bhargave A, Wei C, Cao K (2005) Speech recognition with amplitude and frequency modulations. Proc Natl Acad Sci USA 102:2293-2298. 